\title{
MODELAGEM PARA A ESTIMATIVA DO RENDIMENTO NO DESDOBRO DE TORAS DE Araucaria angustifolia (BERTOL.) KUNTZE
}

\author{
Álvaro Felipe Valério ${ }^{1}$, Luciano Farinha Watzlawick ${ }^{2}$, Rafaelo Balbinot ${ }^{3}$, \\ Marcos Vinicius Wincker Caldeira ${ }^{4}$, Afonso Figueiredo Filho ${ }^{5}$ \\ ${ }^{1}$ Eng. Florestal, Mestrando em Ciências Florestais, UNICENTRO, Irati, PR, Brasil - alvarofvalerio@ yahoo.com.br \\ ${ }^{2}$ Eng. Florestal, Dr., Depto. de Agronomia, UNICENTRO,Guarapuava, PR, Brasil - luciano.watzlawick@pq.cnpq.br \\ ${ }^{3}$ Eng. Florestal, Dr., Depto. de Engenharia Florestal, UNICENTRO, Irati, PR, Brasil - rbalbinot@ yahoo.com.br \\ ${ }^{4}$ Eng. Florestal, Dr., Depto. de Engenharia Florestal, UFES, Alegre, ES, Brasil - caldeiramv@yahoo.com.br \\ ${ }^{5}$ Eng. Florestal, Dr., Depto. de Engenharia Florestal, UNICENTRO, Irati, PR, Brasil - afig@ ufpr.br
}

Recebido para publicação: 11/05/2008 - Aceito para publicação: 14/10/2008

\begin{abstract}
No setor florestal, o prognóstico do estoque e rendimento da matéria-prima, com rapidez e confiabilidade, é de interesse de todo administrador, visto que informações antecipadas podem fornecer subsídios para que a empresa se antecipe e se adapte às novas situações do mercado. $\mathrm{O}$ objetivo deste estudo foi testar e selecionar modelos matemáticos para a predição do rendimento no processo de desdobro de toras de Araucaria angustifolia (Bertol.) Kuntze. O volume foi determinado por Huber. Foram testados 11 modelos, utilizando-se como variável dependente o rendimento em volume de madeira serrada $(\mathrm{R})$, e como variáveis independentes, o diâmetro menor (d), o comprimento $(\ell)$ e o volume total da tora $(\mathrm{v})$. Todas as relações dimensionais foram analisadas via matriz de correlação. A escolha do melhor modelo baseou-se no maior coeficiente de determinação ajustado, no menor erro padrão residual e no comportamento mais uniforme e homogêneo dos valores residuais. O rendimento médio observado foi de 49,2\%, variando entre 42,5 e $57,4 \%$. As maiores correlações foram obtidas para o volume total e o rendimento, bem como entre o diâmetro e o rendimento, de 97,2 e $96,9 \%$, respectivamente. Dentre os modelos testados, o melhor resultado foi obtido pela equação $\mathrm{R}=-0,0040596673+0,0000903028 * \mathrm{~d}^{2}+0,0000146008 *\left(\mathrm{~d}^{2} * \ell\right)$, com precisão $\left(\mathrm{R}_{\mathrm{aj} .}^{2}\right)$ de $96,7 \%$ a um erro padrão $\left(\mathrm{S}_{\mathrm{yx}}\right)$ de $12,8 \%$. Com essa equação, torna-se possível a previsão do rendimento no processo de desdobro, antecipadamente.
\end{abstract}

Palavras-chave: Pinheiro-do-paraná; análise de regressão; serraria.

\begin{abstract}
Modeling to yield estimation in deploying logs of Araucaria angustifolia (Bertol.) Kuntze. The objective of this study was to test and select mathematical models to predict the yield in deploying logs process of Araucaria angustifolia (Bertol.) Kuntze. The volume was determined by Huber. Eleven models were tested using as dependent variable the volume yield of wasting wood (R) and as independent variable the smaller diameter $(\mathrm{d})$, the length $(\ell)$ and the total volume of $\log (\mathrm{v})$. All the dimensional relations were analyzed through correlation matrix. The choice of the best model was based on the highest of adjusted coefficient determination, smallest residual standard error and most uniform behavior and homogeneous of residuals values. The average yield observed was 49,2\% varying from 42,5 to $57,4 \%$. The highest correlations were obtained for total volume and yield, as well as the diameter and yield, 97,2 and 96,9\%, respectively. Among the tested models, the best result was obtained by the equation $\mathrm{R}=-0,0040596673+0,0000903028 * \mathrm{~d}^{2}+0,0000146008 *\left(\mathrm{~d}^{2} * \ell\right)$ with $\left(\mathrm{R}_{\text {aj. }}^{2}\right)$ $96,7 \%$ and standard error $\left(\mathrm{S}_{\mathrm{yx}}\right)=12,8 \%$. With this equation the yield prediction becomes possible in deploying process, in advance.

Keywords: Parana-pine; regression analysis; sawmill.
\end{abstract}




\section{INTRODUÇÃO}

O desenvolvimento de máquinas modernas e equipamentos sofisticados proporcionaram um maior desempenho no processo de transformação da madeira, tornando o mercado mais competitivo e disputado devido à grande capacidade de oferta de produtos acabados a um menor custo. Para as empresas que não acompanharam tal desenvolvimento, esse avanço culminou na redução significativa da margem de lucro para se manter no mercado.

Nesse ambiente competitivo, a informação pode promover o sucesso de uma empresa, fornecendo subsídios que permitam que ela se antecipe e se adapte às novas situações do mercado. Por meio de um planejamento dinâmico, pode-se alcançar o crescimento sustentável, ou, ao contrário, ocorrer a desarticulação de seu plano de crescimento, deixando a organização em situação desfavorável perante outras empresas concorrentes.

No setor florestal, o prognóstico do estoque e rendimento da matéria-prima, com rapidez e confiabilidade, é de interesse de todo administrador. Ele possibilita que decisões sobre desempenho industrial, conjuntura organizacional e uso correto da matéria-prima sejam tomadas antecipadamente às situações adversas, reduzindo gastos e evitando perdas no processo produtivo. Portanto, como os inventários florestais são de grande importância para o planejamento de uma empresa florestal, o conhecimento confiável de seu consumo de matéria-prima e aproveitamento é imprescindível, pois permite ajustar para a indústria a quantidade ideal de madeira a ser consumida, resultando em sua sustentabilidade.

A modelagem apresenta-se como uma ferramenta de grande importância na área florestal, sendo desenvolvida e utilizada para realizar previsões de produtividade dos povoamentos florestais, estimativas hipsométricas, volumétricas e de biomassa (OLIVEIRA; OLIVEIRA, 1988; GUIMARÃES, 1994; LIMA et al., 1996; SCOLFORO, 1997; SCOLFORO, 1998; VEIGA et al., 1998; AZEVEDO et al., 1999; ABREU, 2000; BATISTA et al. 2001; BARROS et al. 2002; CALDEIRA et al. 2002; SILVA et al. 2004; EISFELD et al., 2005; VALÉRIO et al., 2007). Essas ferramentas dendrométricas disponibilizam informações consistentes que auxiliam na quantificação em inventários e na determinação de práticas de manejo corretas, além de servir como importante ferramenta para a tomada de decisões estratégicas em planos de gestão florestal.

O uso de modelos para estimativa do rendimento de serrarias pode gerar informações importantes para a composição de modelos e a formação de sistemas para se conhecer o rendimento futuro de uma floresta em termos de volume de produto final, proporcionando estratégias mais ousadas e seguras, com vistas aos mercados de tais produtos (SOUZA et al., 2007). Porém praticamente existem poucos estudos aplicados ao rendimento em serrarias: Schroeder e Hanks, 1967; Schroeder et al., 1968; Adams; Dunmire, 1977; Yaussey, 1983; Barchet, 2002; Leite, 1994; e Souza et al., 2007. Portanto, apesar de reconhecida a importância do uso de modelos matemáticos no processamento industrial para previsão do rendimento e do volume de resíduos gerados no desdobro e desenrolo de madeira, eles têm sido pouco utilizados no Brasil.

A Araucaria angustifolia (Bertol.) Kuntze, espécie objeto deste estudo, encontra-se em áreas significativas de reflorestamentos com fins econômicos e representa uma importante fonte de matériaprima para a indústria, tendo revelado grande potencial em plantios comerciais (EMBRAPA, 1988; GUERRA et al., 2002).

Diante desse contexto, o presente estudo teve por objetivo ajustar modelos matemáticos para estimar o rendimento no processo de desdobro de toras de Araucaria angustifolia provenientes de reflorestamentos.

\section{MATERIAL E MÉTODOS}

Este estudo foi realizado em duas serrarias fixas convencionais de pequeno porte localizadas nos municípios de Clevelândia e Irati, Paraná. O consumo diário de toras em cada serraria é inferior a $50 \mathrm{~m}^{3}$. Desse modo, conforme a classificação de Rocha (2002), elas se enquadram na categoria pequeno porte, que representa $60 \%$ das serrarias brasileiras.

O processo produtivo é característico da maioria das pequenas empresas madeireiras do país, nas quais o desdobro principal é realizado com uma única serrafita simples vertical, de lâmina larga, 
possuindo um carro porta-toras com três garras fixadoras como sistema de alimentação até o corte, que é executado uma única vez a cada avanço. Posteriormente, o refilamento é feito em uma mesa com uma serra circular simples, e o destopamento com uma serra circular pendular.

As técnicas e características das máquinas possibilitam o desdobro de toras com grande amplitude diamétrica, sendo dispensado um tratamento individualizado para cada tora. Apesar de mais lento, resulta na geração de menor volume de resíduos no processo de conversão da tora. Assim como o processo de desdobro, o fluxograma do sistema produtivo das indústrias é simples (Figura 1).

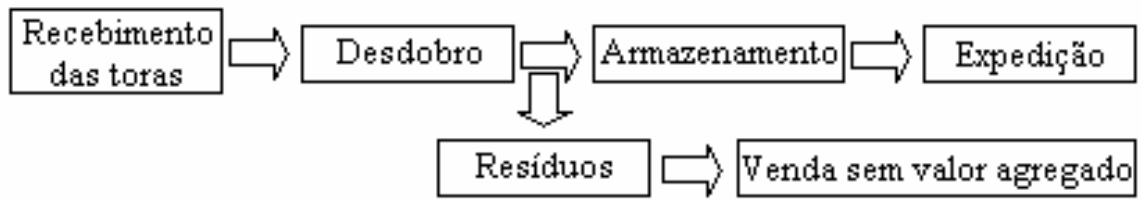

Figura 1. Fluxograma do sistema produtivo.

Figure 1. Flowchart of productive system.

A definição do número de classes de diâmetro foi feita utilizando-se a fórmula de Sturges, que, de acordo com Machado; Figueiredo Filho (2003), é uma das maneiras mais conhecidas para se estabelecer o número de classes para um conjunto de dados. A fórmula é:

$$
\mathrm{N}^{\mathrm{o}} \text { de classes }=1+1,4427 \operatorname{Ln}(\mathrm{n})
$$

Em que:

$\mathrm{n}=$ número de observações disponíveis.

$\mathrm{Ln}=$ logaritmo natural

As toras foram obtidas de árvores plantadas e apresentavam comprimentos entre 2,5 e $5 \mathrm{~m}$. Foram selecionadas de forma aleatória no pátio da empresa e posteriormente classificadas em classes diamétricas, considerando o diâmetro da ponta fina com casca, conforme apresentado na tabela 1.

Tabela 1. Número de toras, diâmetro e comprimento médio por classe diamétrica.

Table 1. Number of logs, diameter and length average per diameter class.

\begin{tabular}{lccc}
\hline Classes diamétricas $(\mathbf{c m})$ & Número de toras & Diâmetro médio $(\mathbf{c m})$ & Comprimento médio $(\mathbf{m})$ \\
\hline$<19$ & 14 & 16,29 & 4,36 \\
$20-24$ & 10 & 22,40 & 4,42 \\
$25-29$ & 11 & 27,06 & 4,07 \\
$30-34$ & 10 & 32,18 & 3,93 \\
$35-39$ & 10 & 37,32 & 3,45 \\
$40-44$ & 9 & 42,28 & 3,83 \\
$45-49$ & 11 & 47,29 & 3,94 \\
$50-54$ & 8 & 52,54 & 3,43 \\
$55-59$ & 9 & 56,87 & 3,91 \\
$60-64$ & 13 & 62,15 & 3,05 \\
$>65$ & 7 & 68,37 & 3,23 \\
\hline Total & 112 & 42,25 & 3,78 \\
\hline
\end{tabular}

O próximo passo foi a marcação dos pontos para a coleta dos diâmetros em duas posições, a ponta de menor diâmetro destinada como variável de entrada para o ajuste dos modelos e a posição central da tora, empregada no método de cubagem de Huber, para quantificação de seu volume real, apresentado, discutido e recomendado por Machado e Figueiredo Filho (2006).

Com a posição central marcada na tora e definida a ponta de menor diâmetro, a medição dupla do diâmetro foi realizada com o auxílio de uma suta. $\mathrm{O}$ volume de cada tora medida foi calculado como segue: 


$$
\mathrm{V}\left(\mathrm{m}^{3}\right)=\left[\left(\frac{\mathrm{Dc}^{2} * \pi}{40000}\right) * 1_{1}\right]
$$

Em que:

$\mathrm{V}=$ volume da tora com casca $\left(\mathrm{m}^{3}\right)$.

$\mathrm{Dc}=$ diâmetro na posição central da tora $(\mathrm{cm})$.

$l_{\mathrm{i}}=$ comprimento da tora $(\mathrm{m})$.

Após o desdobro da tora, o produto resultante foi separado, sendo realizada a medição com o auxílio de trena e paquímetro, e posteriormente a cubagem de cada peça do material processado, obtida com seções de formatos quadrados e retangulares, o que facilitou o trabalho e aumentou a acuracidade na obtenção do volume do material processado. Com a soma das cubagens, determinou-se o volume caracterizado como rendimento, dado em $\mathrm{m}^{3} \cdot \mathrm{tora}^{-1}$, e finalmente, por meio da fórmula apresentada a seguir, o rendimento em percentual foi calculado.

$$
\mathrm{R} \%=\frac{S}{T} * 100
$$

Em que:

$\mathrm{R} \%=$ rendimento em porcentagem.

$\mathrm{S}=$ volume de madeira serrada $\left(\mathrm{m}^{3}\right)$.

$\mathrm{T}=$ volume da tora $\left(\mathrm{m}^{3}\right)$.

Os dados foram organizados e todas as relações dimensionais foram analisadas via matriz de correlação. Por meio de análise de regressão utilizando o método dos mínimos quadrados, testaram-se onze modelos matemáticos (Tabela 2), dos quais, nove lineares e dois não-lineares.

Tabela 2. Modelos testados para estimar o rendimento no processo de desdobro de toras de Araucaria angustifolia.

Table 2. Tested models to estimate the yield in the process of deploying of logs of Araucaria angustifolia.

\begin{tabular}{lc}
\hline Modelo & Formulação matemática \\
\hline 1 & $\mathrm{R}=b_{o} \mathrm{~d}^{b}$ \\
2 & $\mathrm{R}=b_{o}+b_{1} d+b_{2} \mathrm{~d}^{2}$ \\
3 & $\mathrm{R}=b_{o}+b_{1} \mathrm{~d}+b_{2}\left(\mathrm{~d}^{2} \ell\right)$ \\
4 & $\mathrm{R}=b_{o}+b_{1} \mathrm{~d}+b_{2} \mathrm{~d}^{2}+b_{3}\left(\mathrm{~d}^{2} \ell\right)$ \\
5 & $\mathrm{R}=b_{o}+b_{1} \mathrm{~d}^{2}+b_{2}\left(\mathrm{~d}^{2} \ell\right)$ \\
6 & $\mathrm{R}=b_{o}+b_{1} \mathrm{~d}+\mathrm{b}_{2} \ell$ \\
7 & $\mathrm{R}=b_{o} \mathrm{~d}^{b} \ell^{c}$ \\
8 & $\mathrm{R}=b_{o}+b \mathrm{v}$ \\
9 & $\mathrm{R}=b_{o}+b_{1} \mathrm{v}+b_{2} \mathrm{v}^{2}$ \\
10 & $\mathrm{R}=b_{o}+b_{1} \mathrm{v}+b_{2} \mathrm{v}^{2}+b_{3} \mathrm{v}^{3}$ \\
11 & $\mathrm{R}=b_{o}+b_{1} \mathrm{v}+b_{2} \mathrm{v}^{2}+b_{3} \mathrm{v}^{3}+b_{4} \mathrm{v}^{4}$ \\
\hline $\mathrm{R}:$ rendimento $\left(\mathrm{m}^{3}\right) ;$ d: diâmetro menor da tora $(\mathrm{cm}) ; \ell:$ comprimento da tora $(\mathrm{m}) ; \mathrm{v}:$ volume total da tora \\
$\left(\mathrm{m}^{3}\right) ; b_{o}, b_{l}, b_{2}, b_{3}, b_{4}$ : coeficientes de regressão.
\end{tabular}

As estatísticas de ajuste e precisão $\mathrm{R}_{\mathrm{Aj}}^{2} \%, \mathrm{~S}_{\mathrm{yx}} \%$ e Anova da regressão (F) foram calculados para cada caso. A escolha do melhor modelo se baseou nos critérios clássicos de análise de regressão, como o maior coeficiente de determinação ajustado, o menor erro padrão residual, o maior valor de $\mathrm{F}$ da Análise de Variância (Anova) da regressão e o comportamento mais uniforme e homogêneo dos valores residuais. 


\section{RESULTADOS E DISCUSSÃO}

O rendimento médio no desdobro das toras na serraria foi de $49,16 \%$, com consequente perda média de $51,84 \%$. A porcentagem de aproveitamento variou entre toras e entre classes de diâmetro. A tabela 3 apresenta, além das características dendrométricas naturais das toras avaliadas neste estudo, o rendimento semelhante entre as classes analisadas, porém com tendência de maiores rendimentos para as maiores classes de diâmetro.

Tabela 3. Características das variáveis dendrométricas e rendimento médio das toras amostradas de Araucaria angutifolia por classe de diâmetro.

Table 3. Characteristics of dendrometric variables and average yield of logs sampled from Araucaria angutifolia per diameter class.

\begin{tabular}{lcccc}
\hline $\begin{array}{l}\text { Classes diamétricas } \\
(\mathbf{c m})\end{array}$ & $\begin{array}{c}\text { Número de } \\
\text { toras }\end{array}$ & $\begin{array}{c}\text { Diâmetro médio } \\
(\mathbf{c m})\end{array}$ & $\begin{array}{c}\text { Comprimento médio } \\
(\mathbf{m})\end{array}$ & $\begin{array}{c}\text { Rendimento médio } \\
(\mathbf{\%})\end{array}$ \\
\hline$<19$ & 14 & 16,29 & 4,36 & 46,62 \\
$20-24$ & 10 & 22,40 & 4,42 & 42,53 \\
$25-29$ & 11 & 27,06 & 4,07 & 46,72 \\
$30-34$ & 10 & 32,18 & 3,93 & 43,26 \\
$35-39$ & 10 & 37,32 & 3,45 & 50,09 \\
$40-44$ & 9 & 42,28 & 3,83 & 50,03 \\
$45-49$ & 11 & 47,29 & 3,94 & 51,18 \\
$50-54$ & 8 & 52,54 & 3,43 & 52,38 \\
$55-59$ & 9 & 56,87 & 3,91 & 48,63 \\
$60-64$ & 13 & 62,15 & 3,05 & 57,42 \\
$>65$ & 7 & 68,37 & 3,23 & 51,85 \\
\hline Total/média & 112 & 42,25 & 3,78 & 49,16 \\
\hline
\end{tabular}

Essa tendência foi também observada por Biasi (2005) em estudo com espécies tropicais e por Murara Jr. et al. (2005) analisando o rendimento de madeira serrada de Pinus taeda. De acordo com Wade et al. (1992), geralmente o rendimento em madeira serrada aumenta com o incremento do diâmetro das toras, porque o volume de toras perdido com costaneiras e aparas representa menor porcentagem em relação ao volume das toras.

Segundo o Anuário Brasileiro de Economia Florestal (1957), a conversão de toras em tábuas, pranchas, vigas ou outras peças de madeira implica a produção de quantidades distintas de desperdício, de acordo com os fatores que influem no seu volume, como a natureza da matéria-prima, a eficiência das máquinas empregadas pela indústria e as exigências do mercado.

Nesse sentido, Valério et al. (2007), em estudo com Araucaria angustifolia, também observaram que a conicidade, a espessura de casca e o menor diâmetro são características naturais da tora que influenciam no processo de desdobro em serrarias, sendo alguns desses fatores igualmente descritos por outros autores, como Rocha (2002) e Scarnavaca Júnior; Garcia (2003).

A média percentual do rendimento observado foi compatível com os valores encontrados para Araucaria angustifolia por Valério et al. (2007) e observados para outras espécies vegetais em estudos de Iwakiri (1990), Borges et al. (1993), Olandoski et al. (1997), Souza (1997) e Polzl (2003).

Houve uma alta correlação entre as variáveis analisadas neste estudo, exceto para o comprimento das toras. A maior correlação foi encontrada entre o volume total da tora e o rendimento e entre o diâmetro na ponta fina e o rendimento, ambos com correlação linear simples (r) igual a 0,97. A menor correlação foi observada entre o volume da tora e o comprimento $(r=-0,25)$. Essas correlações são apresentadas na tabela 4 . 
Tabela 4. Correlação linear em percentual entre as variáveis analisadas das toras de Araucaria angustifolia.

Table 4. Linear correlation in percentage between analyzed logs variables of Araucaria angustifolia.

\begin{tabular}{lcccc}
\hline Variáveis & Diâmetro $(\mathbf{c m})$ & Comprimento $(\mathbf{m})$ & Volume da tora $\left(\mathbf{m}^{3}\right)$ & Rendimento $\left(\mathbf{m}^{3}\right)$ \\
\hline Diâmetro $(\mathrm{cm})$ & 100 & & & \\
Comprimento $(\mathrm{m})$ & $-46,49$ & 100 & \\
Volume da tora $\left(\mathrm{m}^{3}\right)$ & 95,89 & $\mathbf{- 2 5 , 4 9}$ & 100 & 100 \\
Rendimento $\left(\mathrm{m}^{3}\right)$ & 96,91 & $-37,67$ & 97,16 & 16
\end{tabular}

Diâmetro: diâmetro da ponta fina, em cm; Comprimento: comprimento total da tora, em m; Volume da tora: Volume total da tora, $\mathrm{em} \mathrm{m}^{3}$.

O diâmetro menor da tora apresentou alta correlação com o rendimento, portanto, caracterizou-se como uma variável importante na modelagem, principalmente pela acuracidade e facilidade na sua obtenção, permitindo o desenvolvimento de equações que poderão gerar estimativas do rendimento no desdobro das toras de forma simples, com rapidez e eficiência.

Ao analisar os ajustes obtidos (Tabela 5), nota-se que as equações resultantes dos modelos lineares 4 e 5 apresentaram os melhores resultados, sendo ambas iguais quanto ao erro padrão da estimativa (12,76\%) e aos coeficientes de determinação ajustados (96,7\%). Essa tendência de igualdade no desempenho dos dois modelos também confirmou-se pela análise gráfica da distribuição dos resíduos apresentada na figura 2. Entretanto, para o valor de F da Anova, observou-se um valor mais significativo para o ajuste resultante do modelo $5(1625,93)$ em relação ao modelo $4(1074,21)$.

Tabela 5. Indicadores de ajuste para o rendimento no desdobro de toras de Araucaria angustifolia.

Table 5. Indicators of adjustment to the income in desdobro of logs of Araucaria angustifolia.

\begin{tabular}{lcccc}
\hline $\mathbf{n}$ & $\mathbf{E q u a c ̧ a ̃ o}$ & $\begin{array}{c}\mathbf{R}^{\mathbf{2}} \mathbf{A j .} \\
(\boldsymbol{\%})\end{array}$ & $\begin{array}{c}\text { Syx } \\
(\boldsymbol{\%})\end{array}$ & $\mathbf{F}$ \\
\hline 1 & $\mathrm{R}=-3,763475 * \mathrm{~d}^{1,942339}$ & 94,98 & 14,65 & 2101,04 \\
2 & $\mathrm{R}=-0,030691+0,002117 * \mathrm{~d}+0,000111 * \mathrm{~d}^{2}$ & 95,76 & 14,46 & 1254,97 \\
3 & $\mathrm{R}=-0,115220+0,00625749 * \mathrm{~d}+0,0000189982^{*}\left(\mathrm{~d}^{2} * \ell\right)$ & 95,55 & 14,81 & 1193,69 \\
4 & $\mathrm{R}=-0,006997998+0,0001681332 * \mathrm{~d}+0,000088647 * \mathrm{~d}^{2}+0,0000144949 *\left(\mathrm{~d}^{2} * \ell\right)$ & 96,67 & 12,76 & 1074,21 \\
5 & $\mathrm{R}=-0,0040596673+0,0000903028 * \mathrm{~d}^{2}+0,0000146008 *\left(\mathrm{~d}^{2} * \ell\right)$ & 96,70 & 12,76 & 1625,93 \\
6 & $\mathrm{R}=-0,29324959+0,0117411375 * \mathrm{~d}+0,0221803190 * \ell$ & 94,51 & 16,45 & 957,32 \\
7 & $\mathrm{R}=-4,121265158 * \mathrm{~d}^{2,0253616748 * \ell} \ell^{0,3982902149}$ & 95,65 & 12,93 & 1222,38 \\
8 & $\mathrm{R}=-0,0097369099+0,5201674768 * \mathrm{v}$ & 93,09 & 18,54 & 1496,59 \\
9 & $\mathrm{R}=-0,0140169366+0,54255545358 * \mathrm{v}-0,0188259033 * \mathrm{v}^{2}$ & 93,03 & 18,53 & 1698,89 \\
10 & $\mathrm{R}=0,1346444583-0,0129812349 * \mathrm{v}+0,0005140398 * \mathrm{v}^{2}+-0,0000032511 * \mathrm{v}^{3}$ & 93,08 & 18,38 & 910,96 \\
11 & $\mathrm{R}=0,0783905043-0,0060338603 * \mathrm{v}+0,0002237845 * \mathrm{v}^{2}+0,0000016988^{*} \mathrm{v}^{3}-0,000000296 * \mathrm{v}^{4}$ & 93,03 & 18,36 & 678,10 \\
\hline
\end{tabular}

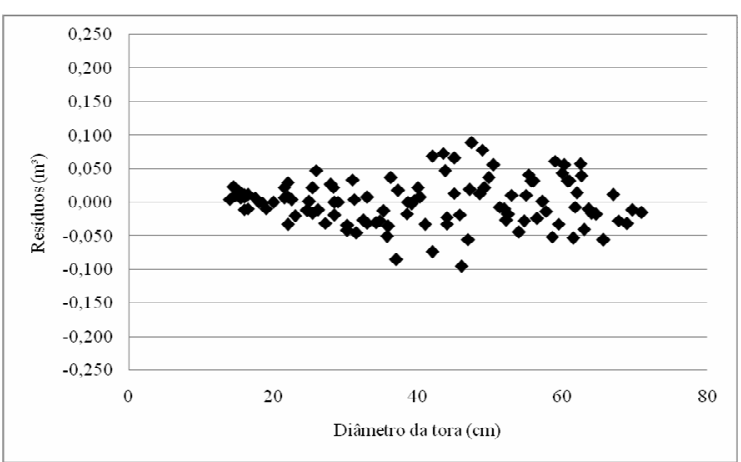

Modelo 4

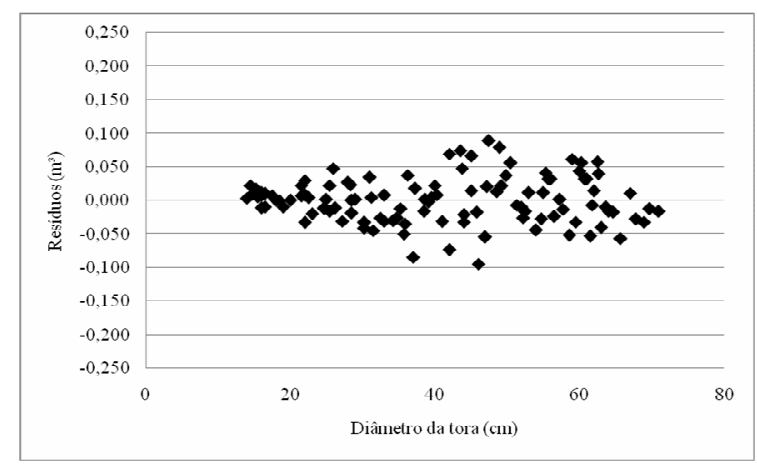

Modelo 5

Figura 2. Distribuição gráfica dos resíduos para o rendimento com os modelos 4 e 5.

Figure 2. Residual distribution for the yield with the models 4 and 5. 
Os resultados obtidos pelo ajuste do modelo 1 apresentaram o maior valor de $\mathrm{F}$ da Anova $(2101,04)$ e uma distribuição homogênea dos valores residuais, semelhante ao modelo 5 de melhor ajuste (Figura 3), bem como um alto coeficiente de determinação corrigido $(94,98 \%)$ e um erro padrão relativamente baixo $(14,65 \%)$. Esses resultados são altamente relevantes, visto que a variável diâmetro representa a única variável independente do modelo, sendo de fácil obtenção e aplicação prática.

As equações resultantes dos modelos 8 a 11, que apresentavam em sua estrutura como variável independente o volume da tora, foram as que proporcionaram os menores coeficientes de determinação ajustados $(93,09 \%, 93,03 \%, 93,08 \%, 93,03 \%)$, os maiores erros padrões de estimativas $(18,54 \%, 18,53 \%$, $18,38 \%, 18,36 \%)$ e, consequentemente, os piores ajustes das análises estatísticas, bem como uma distribuição gráfica de resíduos de maior amplitude, como pode ser observado na figura 4.

$\mathrm{Na}$ prática, o que se observa para a conversão do volume das toras para o volume do rendimento em serrarias é a utilização de um fator percentual médio de rendimento, porém neste estudo se constatou que o maior erro padrão de estimativa $(27,99 \%)$ e o menor coeficiente de determinação $(93,09 \%)$ foram resultado de sua utilização, figurando entre os piores quando comparado com os outros modelos.

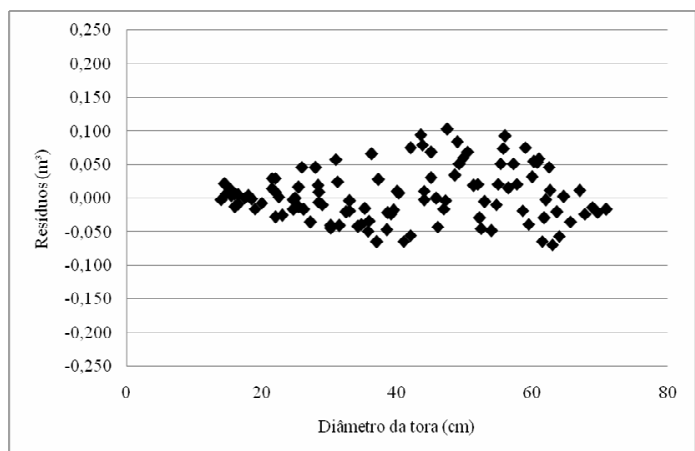

Modelo 1

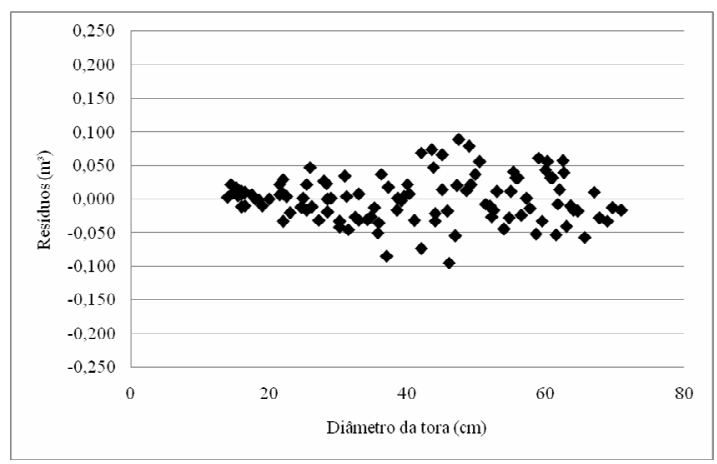

Modelo 5

Figura 3. Distribuição gráfica dos resíduos para o rendimento com os modelos 1 e 5.

Figure 3. Residual distribution for the models 1 and 5.

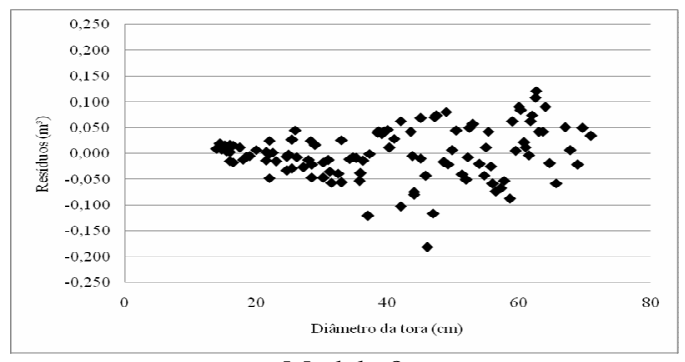

Modelo 8

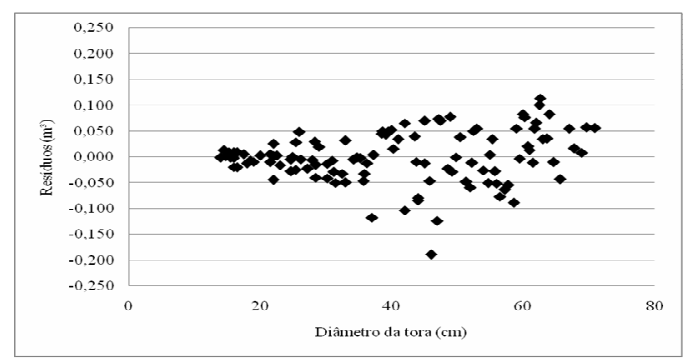

Modelo 10

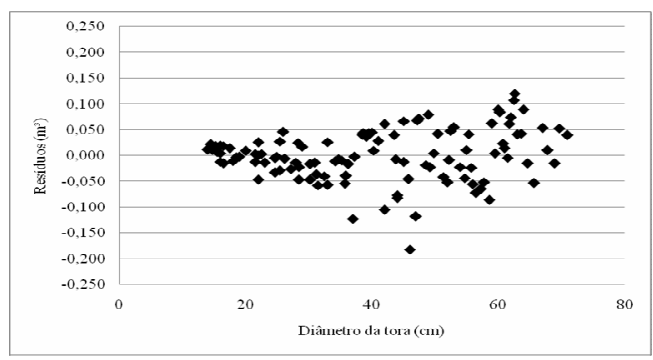

Modelo 9

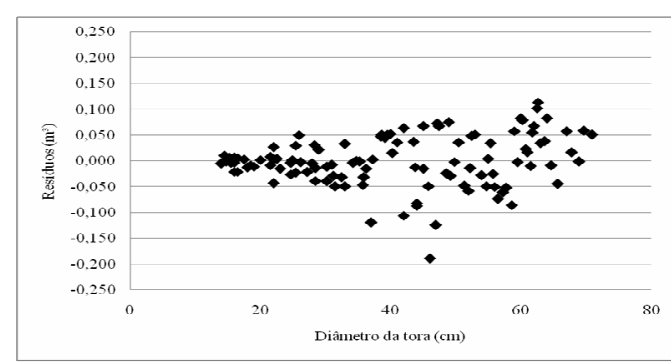

Modelo 11

Figura 4. Distribuição gráfica dos resíduos para o rendimento com os modelos 8 a 11 .

Figure 4. Residual distribution for the models 8 to 11. 
Assim, comparando-se a aplicação do fator de rendimento percentual médio obtido para as toras $(49,16 \%)$ em relação à utilização das técnicas de modelagem, pode-se observar uma melhor distribuição gráfica dos resíduos para o modelo 5 , bem como uma tendência de subestimativa do rendimento no emprego do fator percentual médio para toras com amplitude diamétrica entre 35 e $55 \mathrm{~cm}$ e de superestimativa para toras com diâmetros superiores a $60 \mathrm{~cm}$.

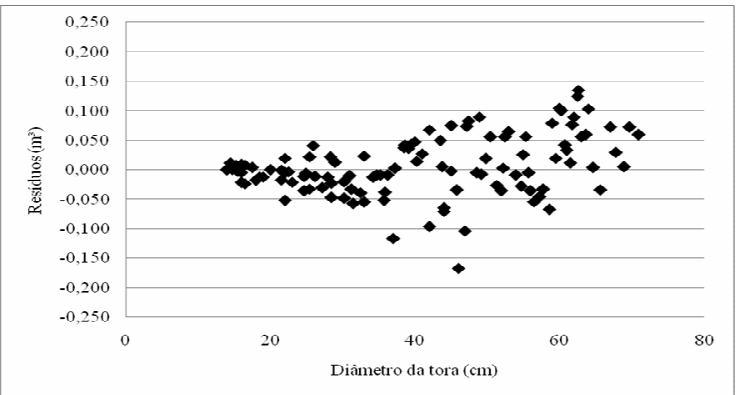

Fator percentual de rendimento $(49,16 \%)$

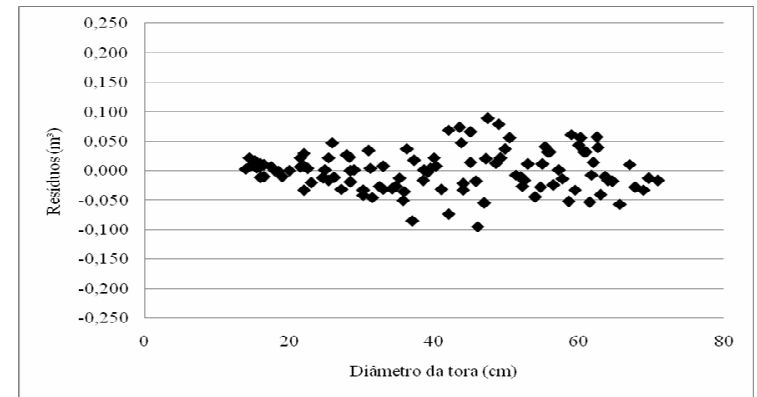

Modelo 5

Figura 5. Distribuição gráfica dos resíduos para o rendimento com a aplicação do fator percentual médio observado e para o modelo (5) de melhor ajuste.

Figure 5. Residual distribution for the yield with application of the average percent factor observed for model of best fitting (5).

Essas análises indicam que a utilização de um fator percentual único e de equações não apropriadas para estimativas do rendimento levam a valores consideravelmente diferentes do real, sendo assim necessário o uso de modelos matemáticos ajustados para cada indústria. Dessa forma, estarão sendo consideradas as características específicas de cada empresa, bem como de cada produto que é processado.

\section{CONCLUSÕES}

- O modelo $5\left(\mathrm{R}=-0,0040596673+0,0000903028 * \mathrm{~d}^{2}+0,0000146008 *\left(\mathrm{~d}^{2} * \ell\right)\right)$ mostrou-se o mais adequado para se estimar o rendimento de madeira serrada de maneira confiável.

- $\mathrm{O}$ modelo $1\left(\mathrm{R}=-3,763475 * \mathrm{~d}^{1,942339}\right)$ apresentou resultados semelhantes ao modelo 5 e tem como variável independente somente o diâmetro menor da tora, o que facilita sua utilização e torna prática a sua aplicação.

- O uso de um fator percentual médio, mesmo que local, pode resultar em estimativas imprecisas do rendimento.

- O desenvolvimento de um modelo matemático local possibilita a predição do rendimento de madeira serrada com grande acuracidade a partir da medição de variáveis fáceis de serem obtidas, gerando informações consistentes e auxiliando no sistema de gerenciamento empresarial.

\section{REFERÊNCIAS}

ABREU, E. C. R. Modelagem para prognose precoce do volume por classe diamétrica para Eucalyptus grandis. 69 f. Dissertação (Mestrado em Ciências Florestais) - Universidade Federal de Lavras, Lavras, 2000.

ADAMS, E. L.; DUNMIRE, D. E. SOLVE II: a technique to improve efficiency ando solve problems in hardwood sawmills. Washington, DC: USDA Forest Service Res. Pap., 1977. 19 p.

ANUÁRIO BRASILEIRO DE ECONOMIA FLORESTAL. Aproveitamento dos resíduos de serraria. Rio de Janeiro: Instituto Nacional do Pinho, v. 9, p. 97-98. 1957.

AZEVEDO, C. P.; MUROYA, K.; GARCIA, L. C.; LIMA, R. M. B.; MOURA, J. B.; NEVES, E. J. M. Relação hipsométrica para quatro espécies florestais em plantio homogêneo e em diferentes idades na Amazônia Ocidental. Boletim de Pesquisa Florestal, Colombo, n. 39, p. 5-29, jul/dez. 1999. 
BARCHET, V. G. Potencialidades de espécies de Eucalyptus para produção de madeira serrada. 166 f. Dissertação (Mestrado em Ciências e Tecnologia da Madeira) - Escola Superior de Agricultura de Luiz de Queiroz, Piracicaba, 2002.

BARROS, D. A.; MACHADO, S. A.; ACERBI JUNIOR, F. W.; SCOLFORO, J. R. S. Comportamento de modelos hipsométricos tradicionais e genéricos para plantações de Pinus oocarpa em diferentes tratamentos. Boletim de Pesquisa Florestal, Colombo, n. 45, p. 3-28. Jul/Dez. 2002.

BATISTA, J. L. F.; COUTO, H. T. Z. do; MARQUESINI, M. Desempenho de modelos de relação hipsométricas: estudo em três tipos de floresta. Scientia Forestalis, Piracicaba, n. 60, p. 149-163. 2001.

BIASI, C. P. Rendimento e eficiência no desdobro de três espécies tropicais. Dissertação - Mestrado. Curso de Pós-graduação em Engenharia Florestal - Universidade Federal do Paraná. Curitiba, 2005. 61 p.

BORGES, A. S.; GINIGLIO, G.; BRITO, J. O. Considerações energéticas e econômicas sobre resíduos de madeira processada em serraria. CONGRESSO FLORESTAL PANAMERICANO, 1.; CONGRESSO FLORESTAL BRASILEIRO, 7., 1993, Curitiba. Anais... São Paulo: Sociedade Brasileira de Silvicultura: Sociedade Brasileira de Engenheiros Florestais, 1993. v.3. p. 603-606.

CALDEIRA, M. V. W.; SCHUMACHER, M. V.; SCHEEREN, L. R.; BARICHELLO, L. R.; WATZLAWICK, L. F. Relação hipsométrica para Acacia mearnsii com diferentes idades. Boletim de Pesquisa Florestal, Colombo, n. 45, p. 57-68, jul/dez. 2002.

EISFELD, R. L.; SANQUETA, C. R.; ARCE, J. E.; MAESTRI, R.; WEBER, K. S. Modelagem do crescimento e da produção de Pinus taeda L. por meio de função probabilística. Floresta, Curitiba, p. 317-328, v. 35, n. 2, 2005.

EMBRAPA. Centro Nacional de Pesquisas de Florestas, Colombo, PR. Manual técnico da bracatinga (Mimosa scabrella Benth.). Colombo, 1988. 70p. (Documentos, n.20).

GUERRA, M. P.; SILVEIRA, V.; REIS, M. S.; SCHNEIDER, L. Exploração, manejo e conservação da araucária (Araucaria angustifolia). In: Sustentável Mata Atlântica: a exploração de seus recursos florestais. São Paulo: SENAC. 2002.

GUIMARÃES, D. P. Desenvolvimento de um modelo de distribuição diamétrica de passo invariante para prognose e projeção da estrutura de povoamentos de eucalipto. 160f. Tese (Doutorado em Ciência Florestal) - Universidade Federal de Viçosa, Viçosa, MG, 1994.

IWAKIRI, S. CPPF - Centro de Pesquisa de Produtos Florestais. Série Técnica no. 08. Sobre a LIMA, J.C. de. Custos (Cálculos, Sistemas e Análises). 2 2a Edição, Editora Atlas. NÃO SEI O QUE É ISTO

LEITE, H. G. Conversão de troncos em multiprodutos da madeira, utilizando programação dinâmica. 230 f. Tese (Doutorado) - Universidade Federal de Viçosas, Viçosa, MG,1994.

LIMA, P. C. F.; OLIVEIRA, E. B.; MACHADO, S. A. Equações para estimativa de biomassa de espécies de Prosopis no semi-árido brasileiro. Boletim de Pesquisa Florestal, Colombo, n. 32/33, p. 67-79. Jan/Dez. 1996.

MACHADO, S. A.; FIGUEIREDO FILHO, A. Dendrometria. 2. ed. Guarapuava. Universidade Estadual do Centro Oeste. UNICENTRO, 2006. 316 p.

MURARA JR, M. I.; ROCHA, M. P.; TIMOFEICZYK JR, R. Rendimento em madeira serrada de Pinus taeda para duas metodologias de desdobro. Revista Floresta, Curitiba, v. 35, n. 3, set/dez. 2005.

OLANDOSKI, D. P.; BRAND, M. A.; GORNIAK, E. Avaliação do rendimento, quantidade, qualidade e aproveitamento de resíduos no desdobro de Pinus spp. In: UNIVERSIDADE FEDERAL DO PARANÁ. Evento de Iniciação Científica, 5., 1997, Curitiba. Anais... Curitiba, 1997. p. 379.

OLIVEIRA, Y. M. M. de; OLIVEIRA, E. B. de. Relação hipsométrica para bracatinga. In: EMBRAPA. Centro Nacional de Pesquisas Florestais. Manual técnico da bracatinga (Mimosa scabrella Benth.). Curitiba: Embrapa-CNPF, 1988, p. 46-47. (Documentos, n. 20). 
POLZL, W. B.; SANTOS, A. J. dos; TIMOFEICZYK JR, R.; POLZL, P. K. Cadeia produtiva do processamento mecânico da madeira - segmento da madeira serrada no Estado do Paraná. Floresta,Curitiba, n. 33(2), 2003, p. 127-134.

ROCHA, M. P. Eucalyprus grandis Hill ex Maiden e Eucalyptus dunnii Maiden como fontes de matéria prima para serrarias. 185 f. Tese (Doutorado em Engenharia Florestal) - Setor de Ciências Agrárias, Universidade Federal do Paraná. Curitiba. 2000.

ROCHA, M. P. Técnicas e Planejamento em Serrarias. Edição revisada. Curitiba, 2002.121 p.

SCARNAVACA JUNIOR, L.; GARCIA, J. N. Rendimento em madeira serrada de Eucaliptus uroplhylla. Scientia Forestalis, Piracicaba, n. 63, p. 32-43, 2003.

SCOLFORO, J. R. S. Técnica de regressão aplicada para estimar: volume, biomassa, relação hipsométrica e múltiplos produtos de madeira. Lavras: ESAL: FAEPE, 1997. 292 p.

SCOLFORO, J. R. S. Modelagem do crescimento e da produção de florestas plantadas e nativas. Lavras: UFLA/FAEPE, 1998, 441 p.

SCHROEDER, J. G.; CAMPBELL, R. A.; RODENBACH, R. C. Southern pine log grades for yard and structural lumber. Washington, DC: USDA Forest Service Res. Pap., 1968. 9 p.

SCHROEDER, J. G.; HANKS, L. F. Lumber grade yields for factory grade northern red oak sawlogs. Washington, DC: USDA Forest Service Res. Pap., 1967. 7 p.

SILVA, H. D.; FERREIRA, C. A.; CORRÊA, R. S.; BELLOTE, A. F. J.; TUSSOLINI, E. L. Alocação de biomassa e ajuste de equações para estimativa de biomassa em compartimentos aéreos de Eucalyptus benthamii. Boletim de Pesquisa Florestal, Colombo, n. 49, p. 83-95, jul/dez. 2004.

SOUZA, M. R. Tecnologias para usos alternativos de resíduos florestais. Experiências do laboratório de produtos florestais - IBAMA, na área de utilização de resíduos florestais e agrícolas. In: WORKSHOP SUL-AMERICANO SOBRE OS USOS ALTERNATIVOS DE RESÍDUOS DE ORIGEM FLORESTAL E URBANA, 1997. p. 49-70.

SOUZA, A. N.; OLIVEIRA, A. D.; SCOLFORO, J. R. S.; MELlO, J. M.; CARVALHO, L. M. T. Modelagem do rendimento no desdobro de toras de Eucalipto cultivado em sistema agroflorestal. Cerne, Lavras, v. 13, n. 2, p. 222-238, abr./jun. 2007.

VALÉRIO, A. F.; SANTOS, R. T.; SILVESTRE, R.; KOEHLER, H. S.; WATZLAWICK, L. F. Ajuste de modelos matemáticos para estimativa de biomassa nos distintos componentes de uva do japão (Hovenia dulcis Thunb.). Ambiência, Guarapuava, v. 3, n. 3, p. 325-336, 2007.

VALERIO, A. F.; WATZLAWICK, L. F.; SANTOS, R. T.; BRANDELERO, C.; KOEHLER, H. S. Quantificação de resíduos e rendimento no desdobro de Araucaria angustifolia (Bertol.) Kuntze. Floresta, Curitiba, v. 37, n. 3, p. 387-398, 2007.

VEIGA, R. A. de A.; CARVAlho, C. M. de; BRASIL, M. A. M. Determinación de equaciones de volumen y peso para arboles de Acacia mangium Willd. Y Eucalyptus grandis Hill ex- Maiden ocasion del primer corte. In: CONGRESO LATINOAMERICANO IUFRO, 1., 1998, Valdivia, Chile. EI manejo sustentable de los recursos forestales, desafio del siglo XXI: actas. [S.I.]: CONAF; [Viena]: IUFRO, 1998. Resumo.

YAUSSEY, D. A. Multivariate regression model for predicting lumber grade volumes of northern red oak sawlogs. Washington, DC: USDA Forest Service, 1983. 30 p.

WADE, M. W.; BULlARD, S. H.; STEELE, P. H.; ARAMAN, P. A. Estimating hardwoord sawmil conversion efficiency based on sawing machine and log characteristics. Forest Products Journal, Madison, v. 42. n. 11/12. p. 21-26. 1992. 